\title{
POSSIBLE CLUES CONCERNING THE ADVERSE RELATIONSHIP BETWEEN DAIRY COW FERTILITY AND NEGATIVE ENERGY BALANCE
}

\author{
Jorritsma R, Rubio Pomar F.J., Bevers M.M., Kruip T.A.M., Noordhuizen J.P.T.M., Wensing, Th. \\ Dept of Farm Animal Health, Ruminant Health Unit, Faculty of Veterinary Medicine, Utrecht University
}

\begin{abstract}
High milk production in dairy cows is frequently associated with diminished fertility. Among other factors that are either prerequisites for or enhancing fertility, there is increasing evidence that also effects of high milk production play a role. More specific, the degree of adaptation to the shortage of energy in early lactation seems responsible for the fertility changes. The adaptation is reflected in concentrations of glucose, non-esterified fatty acids (NEFA) and insulin in blood and the amount of triacylglycerol (TAG) accumulated in the liver.
\end{abstract}

To address the consequences of non-adaptation, we showed that there are detrimental effects of NEFA on in vitro performance of granulosa cells. Also, higher amounts of TAG in the liver are associated with prolonged intervals between parturition and first heat and between parturition and pregnancy.

A final evaluation of the effects of non-adaptation on fertility can not rely on an univariate approach of the situation only. For this reason, an animal model was designed, which enables frequent monitoring during the period of adaptation. The model consists of overfeeding during the dry period and restricted feeding during the first days of lactation to provoke differences in post partum negative energy balance between cows. This will result in differences in adaptation. It has been shown that nonadaptation coincides with deviated concentrations of glucose and insulin in blood and an increased intensity of lipolysis and fatty acid supply to the liver.

The first study, using the animal model, focussed on the effects of non-adaptation on oocyte quality. The quality of the oocyte was assessed by investigating its developmental potency. Therefore, collected immature oocytes were in vitro matured and the rate of blastocyst formation was assessed. Secondly, the effects of non-adaptation were measured on the quality of the obtained blastocysts by measuring the degree of apoptosis and the localization of the apoptotic cells.

Preliminary results show, that apoptosis in the inner cell mass was more frequently present in blastocysts from non-adapted compared to adapted cows. 